\title{
Space Astrometry JASMINE
}

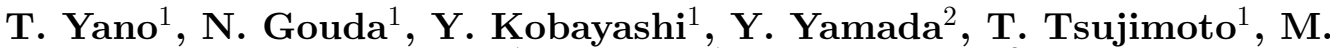 \\ Suganuma $^{1}$, Y. Niwa ${ }^{1}$, M. Yamauchi ${ }^{3}$ \\ ${ }^{1}$ National Astronomical Observatory, Mitaka, Tokyo 181-8588, Japan \\ email: yano.t@nao.ac.jp, naoteru.gouda@nao.ac.jp, yuki@merope.mtk.nao.ac.jp, \\ taku.tsujimoto@nao.ac.jp, suganuma@merope.mtk.nao.ac.jp, kazin.niwa@nao.ac.jp \\ ${ }^{2}$ Graduate School of Science, Kyoto University, Sakyo-ku, Kyoto 606-8502, Japan \\ email: yamada@scphys.kyoto-u.ac.jp \\ ${ }^{3}$ Department of Astronomy, School of Science, University of Tokyo, Tokyo 113-0033, Japan \\ email: yamauchi@merope.mtk.nao.ac.jp
}

\begin{abstract}
JASMINE is the acronym of the Japan Astrometry Satellite Mission for INfrared (zband :0.9 micron) Exploration, and is planned to be launched around 2017. The main objective of JASMINE is to study the fundamental structure and evolution of the Milky Way bulge components. In order to accomplish these objectives, JASMINE will measure trigonometric parallaxes, positions and proper motions of about ten million stars in the Galactic bulge with a precision of 10 microarcsec at $\mathrm{z}=14 \mathrm{mag}$.

The primary mirror for the telescope has a diameter of $75 \mathrm{~cm}$ with a focal length of $22.5 \mathrm{~m}$. The back-illuminated CCD is fabricated on a 300 micron thick substrate which is fully depleted. These thick devices have extended near infrared response. The size of the detector for z-band is $3 \mathrm{~cm} \times 3 \mathrm{~cm}$ with $2048 \times 2048$ pixels. The size of the field of view is about $0.6 \mathrm{deg} \times 0.6 \mathrm{deg}$ by using 64 detectors on the focal plane. The telescope is designed to have only one field of view, which is different from the designs of other astrometric satellites. JASMINE will observe overlapping fields without gaps to survey a total area of about $20 \mathrm{deg} \times 10 \mathrm{deg}$ around the Galactic bulge. Accordingly we make a "large frame" of $20 \mathrm{deg} \times 10 \mathrm{deg}$ by linking the small frames using stars in overlapping regions. JASMINE will observe the Galactic bulge repeatedly during the mission life of about 5 years.
\end{abstract}

Keywords. space vehicles, astrometry, Galaxy: bulge

\section{Summary of the observational procedures}

The procedures of our method for measuring astrometric parameters of Galactic bulge stars can be summarize in the following three steps.

\section{Centroiding of stars}

The centroids of stars in a field of view are determined using a photon weighted means of stars Yano et al. (2004) Yano et al. (2006). Consequently positions of stars in the field of view (hereafter we call small frame) are obtained.

\section{Construction of a large frame}

JASMINE will take overlapping fields of view without gaps to survey the area of about $20^{\circ} \times 10^{\circ}$. During this time (about $10 \mathrm{~h}$ ) variations or distortions of the optical equipments must be small (order of $10 \sim 100 \mathrm{pm}$ ). In order to confirm the stability of the optical equipments, they are monitored using a laser interferometer. Then, we connect all small frames using positions of stars in the overlapping areas to form a large region (hereafter large frame).

\section{Estimating the parallaxes of stars}

We continue the above procedure about $10^{4}$ times during the mission life. We derive the astrometric parameters such as parallaxes of stars using the large frames. In connecting 
all large frames we make use of QSOs as fixed points. As a consequence we obtain the positions of stars directly into the International Celestial Reference System (ICRS).

\section{QSO}

We plan to make use of QSOs in order to obtain astrometric parameters and fix to ICRS (International Celestial Reference System). There are many bright QSO candidates in the survey area of JASMINE. There are over 50 candidates in the survey area, and there are 14 candidates brighter than $\mathrm{K} \leqslant 13$. These candidates are selected using the photometric variability during a few years in OGLE (Optical Gravitational Lensing Experiment). Although we do not know how many candidates are QSOs, we expect that about 40-70 $\%$ of candidates are QSOs. Up to now one bright QSO is confirmed in the survey area.

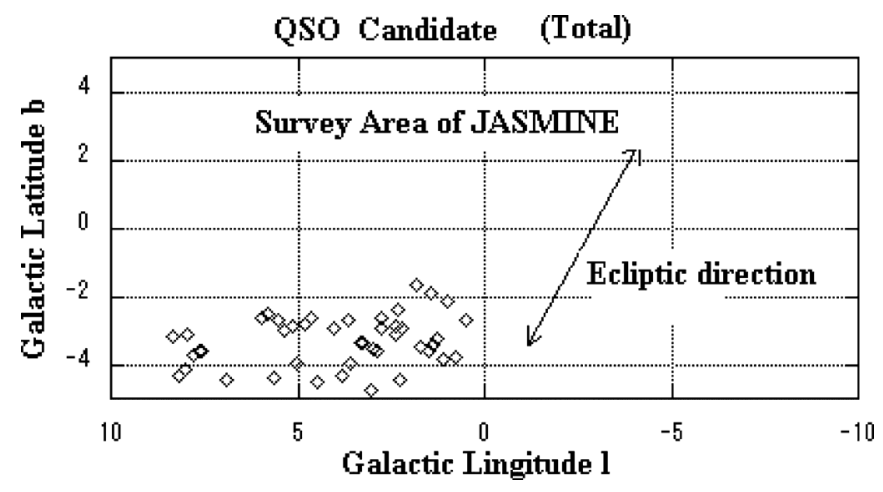

Figure 1. QSO candidates in the survey area.

\section{Thermal environment}

In order to satisfy the objective of high accuracy measurement, the optical equipment (optics, detectors, and so on ) must be stabilized. $\mathrm{SiC}$ is one of the candidates for material of optics (mirrors and structures). In this case the thermal capacity decreases as the temperature decreases. The expansion coefficient also decreases as the temperature decreases. The effect of the thermal expansion exceeds that of the thermal capacity. As a consequence, optical equipment is stabilized as the temperature decreases. However the values of the thermal expansion at low temperatures are uncertain. Then it is important to investigate thermal expansion of materials at low temperature.

\section{References}

Yano, Taihei, Gouda, Naoteru, Kobayashi, Yukiyasu, Tsujimoto, Takuji, Nakajima, Tadashi, Hanada, Hideo, Kan-ya, Yukitoshi, Yamada, Yoshiyuki, Araki, Hiroshi, Tazawa, Seiichi, Asari, Kazuyoshi, Tsuruta, Seiitsu, \& Kawano, Nobuyuki 2004, PASP, 116, 667

Yano, Taihei, Araki, Hiroshi, Gouda, Naoteru, Kobayashi, Yukiyasu, Tsujimoto, Takuji, Nakajima, Tadashi, Kawano, Nobuyuki, Tazawa, Seiichi, Yamada, Yoshiyuki, Hanada, Hideo, Asari, Kazuyoshi, \& Tsuruta, Seiitsu 2006, PASP, 118, 1448 